\title{
Clinicopathological Features of Primary Jejunoileal Tumors
}

\author{
Chang Hyun Kim, Bong Hyeon Kye, Jae Im Lee, Soo Hong Kim, Hyung Jin Kim, Won Kyung Kang, \\ Seong Taek Oh \\ Department of Surgery, The Catholic University of Korea School of Medicine, Seoul, Korea
}

Purpose: Tumors of the small bowel are rare, accounting for about 3-6\% of all gastrointestinal neoplasms, though they cover more than $90 \%$ of the intestinal surface. However, diagnosis and treatment are difficult and present an ongoing challenge for both gastrointestinal surgeons and gastroenterologists. The aim of this study was to investigate the clinical features of small bowel tumors.

Methods: Between November 1994 and November 2007, 81 patients underwent treatments for primary tumors in the jejuno-ileal region at the Department of Surgery, Kangnam St. Mary's Hospital, the Catholic University of Korea. A retrospective review of the patients' characteristics and variable tumor factors was performed.

Results: The mean age of the patients was 53.2 years with 48 men and 33 women. The most common symptom was abdominal pain (59.3\%), followed by bleeding (22.2\%) and an abdominal mass (6.2\%). We found that the patients with ileal tumors complained mainly of abdominal pain (72.9\%) whereas the patients with jejunal tumors presented with gastrointestinal bleeding (36.4\%) $(\mathrm{P}=0.048)$. Seventy-six of the 81 patients $(93.8 \%)$ had malignant tumors, including $40(49.4 \%)$ gastrointestinal stromal tumors, 26 (32.1\%) lymphomas and $5(6.2 \%)$ adenocarcinomas. No postoperative mortalities were observed. The overall 5-year survival rate of the patients with malignant small bowel tumors was $31.8 \%$.

Conclusion: Because the clinical features of a primary tumor of the small bowel are obscure and its diagnosis is difficult, maintaining a high degree of suspicion and recognizing the possibility of a primary small bowel tumor are important.

\section{Keywords: Small intestine; Gastrointestinal stromal tumors; Lymphoma}

\section{INTRODUCTION}

The small intestine represents $90 \%$ of the entire surface of the gastrointestinal tract, and $75 \%$ of its length. However, tumors rarely develop in the small intestine, showing an incidence of $5 \%$ forf all tumors in the gastrointestinal tract, and among them, malignant tumors represent only $1-2 \%$ of the total [1]. Histologically, of the small intestine tumors, more than $60 \%$ are malignant tumors, and among them, the incidences of gastrointestinal interstitial tumors, adenocarcinomas, malignant lymphomas, and carcinoids have been reported to be high [2].

Received: June 20, 2010 Accepted: September 20, 2010

Correspondence to: Seong Taek Oh, M.D.

Department of Surgery, The Catholic University of Korea School of Medicine, Banpo 4-dong, Seocho-gu, Seoul 137-701, Korea

Tel: +82-2-2258-6100, Fax: +82-2-595-2822

E-mail: stoh@catholic.ac.kr

(C) 2010 The Korean Society of Coloproctology

This is an open-access article distributed under the terms of the Creative Commons Attribution NonCommercial License (http://creativecommons.org/licenses/by-nc/3.0) which permits unrestricted noncommercial use, distribution, and reproduction in any medium, provided the original work is properly cited.
Since small intestine tumors have a different prognosis and since the appropriate surgical and medical treatments are based on their histological diagnosis, selection of treatment methods through an accurate diagnosis is important [3]. Even though cases with an abdominal mass, hemorrhage, or peritonitis caused by an intestinal perforation can be easily diagnosed by clinical presentation, physical examination, and imaging studies, the diagnosis for tumors of the small intestine is usually difficult because most of them have nonspecific clinical symptoms and nonspecific physical examination results compared to other tumors. In addition, because of the anatomical location of the small intestine, excluding the proximal duodenal area, a direct approach is difficult; thus, in spite of the development of diagnostic techniques, the diagnosis rate is not high [4]. Therefore, when such a tumor is diagnosed, it is usually accompanied by complications or is far advanced, showing a poor prognosis [5].Therefore, in this study, we analyzed the clinical characteristics of and the treatment methods, and prognoses for patients with primary small bowel tumors and tried to find differences based on clinical presentations, physical examination results, and histological findings. 


\section{METHODS}

Eighty one patients were treated for primary small bowel tumors in the Department of Surgery, Seoul Saint Mary's Hospital, the Catholic University of Korea, from November 1994 to November 2007. Age, gender, primary tumor site, clinical symptoms and signs, surgery methods, prognosis, histological findings, and other clinical results were retrospectively compared and analyzed. Primary small bowel tumors were considered, tumors that developed in the duodenum were excluded, and only tumors that developed in the jejunum and the ileum were analyzed. In regard to the gastrointestinal stromal tunor (GIST), according to the AJCC classification, Stage I cases were considered as benign, and cases higher than Stage II were classified as having malignant potentials. Most patients were admitted to the hospital with abdominal symptoms. Diagnostic preoperative studies included CT, MRI, PET-CT and endoscopy. Eleven non-diagnosed patients underwent a diagnostic laparotomy.

For statistical analysis, the chi-square test was used for categorical variables. The survival rate was analyzed by using the Kaplan-Meier method. P values less than 0.05 were considered to be significant.

\section{RESULTS}

\section{Gender and age}

Among the 81 patients, 48 were male and 33 were female, males being more prevalent. The mean age was 53.2 years (range, 2 to 84 years), and 38 patients ( $46.9 \%$ ) were in the 5th decade, which showed the highest frequency.

\section{Pathohistological findings and the distribution of the primary tumor site}

A review of the pathohistological findings of small bowel tumors showed that, among the 81 cases, $25.9 \%$ were benign, and $74.1 \%$ were malignant. Their pathological classifications

Table 1. Histologic findings of tumors

\begin{tabular}{lc}
\hline Pathology & Number \\
\hline Benign & 21 \\
Gastrointestinal stromal tumor & 16 \\
Inflammatory fibroid tumor & 3 \\
Adenomyoma & 1 \\
Adenoma & 1 \\
Malignant & 60 \\
Gastrointestinal stromal tumor & 24 \\
Lymphoma & 26 \\
Adenocarcinoma & 5 \\
Leiomyosarcoma & 3 \\
Carcinoid & 1 \\
Neuroendocrine tumor & 1 \\
\hline
\end{tabular}

are shown in Table 1. Among the 81 cases, primary tumors in the jejunum were observed in 33 cases (40.7\%), and tumors were onserved in the ileum in 48 cases (59.3\%). An examination of the correlation of primary tumor site with histological diagnostic distribution showed that the gastrointestinal stromal tumors were found preferentially in the jejunum (75.8\%) and that malignant lymphomas were found preferentially in the ileum (50\%) $(\mathrm{P}=0.001)$.

\section{Clinical symptoms and physical examination findings}

The major symptoms of the patients at the time of admission were 48 cases of abdominal pain (59.3\%), 18 cases of hemorrhage (22.2\%), and 5 cases of abdominal mass (6.2\%). In addition, 10 patients (12.3\%) complained of constipation, vomiting, vertigo, and diarrhea. An examination of the correlation of the primary tumor site with the major symptoms of the patients showed that abdominal pain and hemorrhage had similar frequencies of occurrence (39.3\% vs. $36.4 \%$ ) in tumors that developed in the jejunum and that abdominal pain had the highest frequency of occurrence $(72.9 \%)$ in tumors that developed in the ileum $(\mathrm{P}=0.048)$ (Fig. 1).

When the symptoms are related to histological classification, in gastrointestinal stromal tumors, abdominal pain was observed in 14 cases and hemorrhage in 10 cases. Patients with a malignant lymphoma showed symptoms such as abdominal pain, abdominal mass, and fever, or they were asymptomatic. Among the patients with a malignant lymphoma, abdominal pain appeared in 19 cases, being the most prevalent. Among patients with abdominal pain as the chief complaint, intestinal perforation or intussusceptions, as diagnosed by using abdominal computed tomography or explo-laparatomy, were observed in 3 cases and 6 cases, respectively.

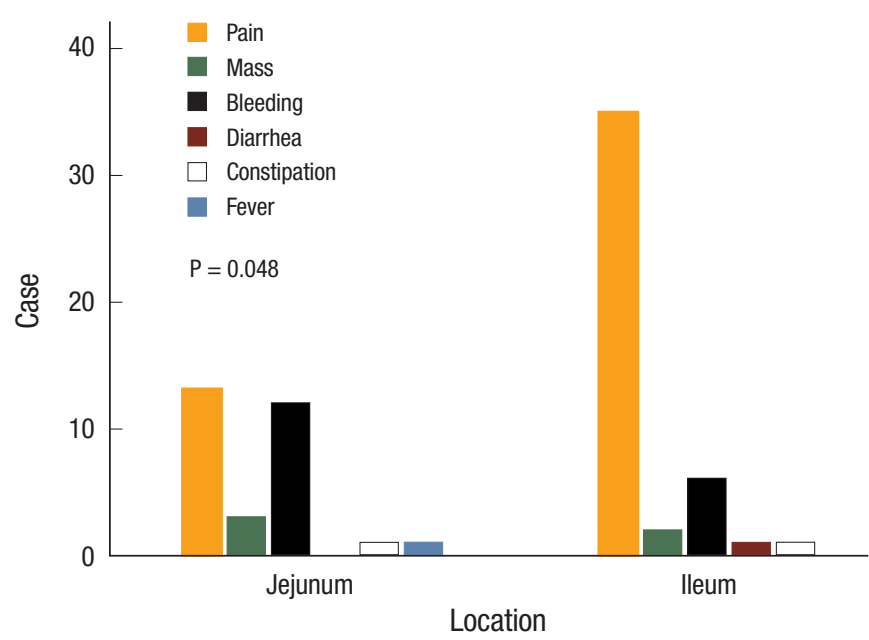

Fig. 1. Main Symptoms of primary small bowel tumors are significantly different between those in the jejunum and those in the ileum. 


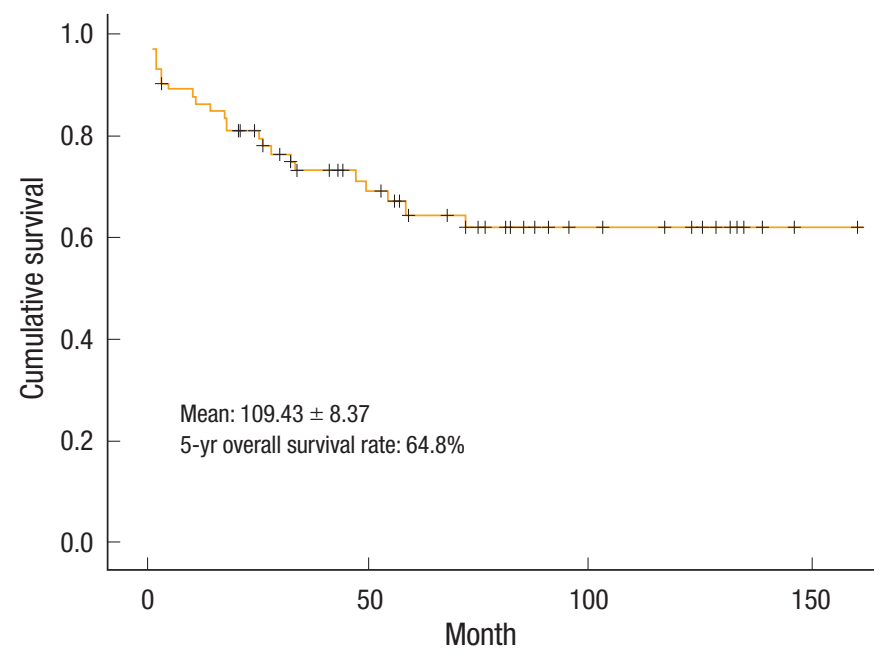

Fig. 2. Overall survival in patients with malignant small bowel tumors.

\section{Preoperative diagnosis}

For pre-surgical diagnosis, abdominal computed tomography, upper gastrointestinal imaging, abdominal ultrasonography, or endoscopic examination were used in most cases. Most small bowel tumors were diagnosed by using abdominal computed tomography (44 cases), endoscopic examination (11 cases), and abdominal ultrasonography (5 cases). For 11 cases which were not diagnosed by any tests, an explo-laparotomy was performed.

\section{Surgical methods}

Among the 81 patients, a radical resection was performed in 75 cases, a resection and anastomosis in 69 cases $(85.1 \%)$ and a wedge resection in 6 cases (7.4\%); a bypass or other resection was performed in 6 cases.

\section{Outcomes and prognosis}

Examining the postsurgical outcome of our patients none died of postsurgical complications. Among 26 lymphoma patients, postoperative radiation therapy and chemotherapy were performed on 22 patients, and chemotherapy was performed on 1 carcinoid patient, 1 neuroendocrine tumor patient and 11 gastrointestinal stromal tumor patients.

The mean follow-up observation period of the 74 malignant tumor patients was 54.7 months, and the mean survival time and the 5-year survival rate were observed to be $109.43 \pm 8.3$ months and $64.8 \%$, respectively (Fig. 2). In the analysis of the survival rate according to histological diagnosis, the 5-year survival rate of patients with gastrointestinal stromal tumors $(82.5 \%)$ was observed to be statistically significantly higher than that for patients with a carcinoid $(40 \%)$ or with a malignant lymphoma (46.4\%) $(\mathrm{P}=0.0007)$ (Fig. 3).

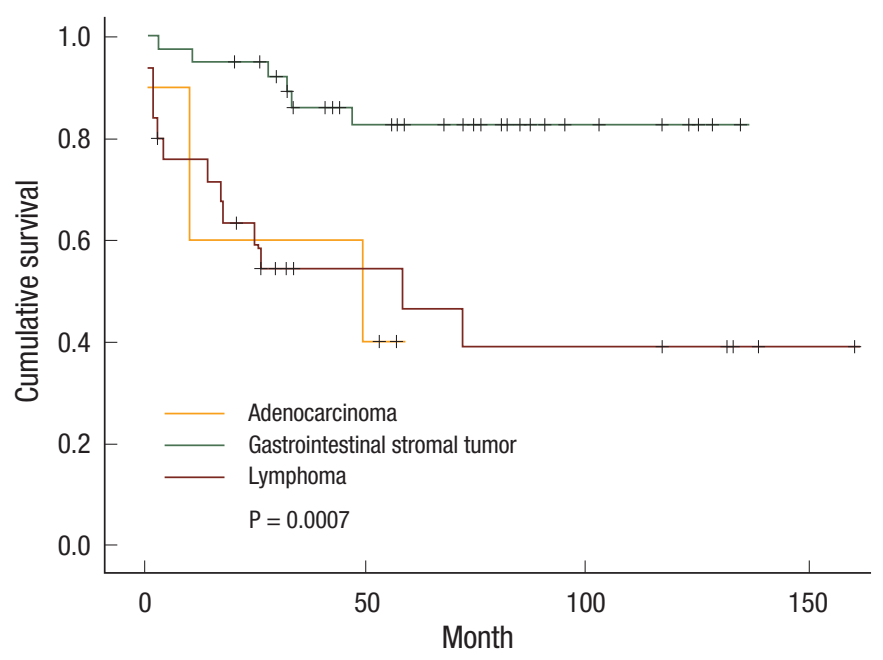

Fig. 3. Overall survivals according to the histology of the primary small bowel tumors are significantly different.

\section{DISCUSSION}

Primary small bowel tumor is a very rare disease. Although the small intestine represents of $90 \%$ of the entire gastrointestinal surface and $75 \%$ of its length, tumors developing in the small intestine are $5 \%$ of all tumors developing in the gastrointestinal tract, and among them, malignant tumors are merely $1-2 \%$ [1]. The cause of carcinogenesis is not clear. Nonetheless, the incidence of malignant tumors has been reported to be particularly low in the small intestine because of the rapid regeneration and proliferation of the small intestine mucosa, and this lowers the sensitivity to cancer cells. Unlike the large intestine, in the small intestine, the number of bacteria is small, and carcinogens due to bacterial metabolites are generated less. In addition, the rate of food transit in the small intestine is rapid, and the time of contact with carcinogens is short, benzopyren hydroxylase, which neutralizes carcinogens, being abundant in the small intestine. Lastly, lymph node tissues and the concentration of IgA are high in the ileum, which protects the small intestine from viruses and suppresses tumor cell growth [6-8].

The distensibility of the small intestine is good, and the content is liquid, so clinical symptoms of small bowel tumors are nonspecific and manifest slowly. Because of these characteristics, unless severe symptoms are shown, most cases are difficult to diagnose, and the disease is advanced when diagnosed in many cases [1]. Although most small bowel tumors are asymptomatic, malignant tumors are known to show more symptoms than benign tumors. Darling et al. [9] have reported that 75\% of small bowel tumors are benign; however, $75 \%$ of the patients on whom surgery had been performed due to symptoms had malignant tumors. Naef et al. [10] have reported that the most 
frequent clinical symptoms are abdominal pain, anemia, weight loss, and hemorrhage. Catena et al. [4] reported the clinical features of small bowel tumors that manifested during emergency conditions requiring surgery, and intestinal obstruction was pointed out to be the most common symptom. In addition, intestinal perforation and intestinal hemorrhage were reported.

An analysis of the clinical symptoms according to histological findings revealed that gastrointestinal stromal tumors show hemorrhage, carcinoids showed bowel obstruction, malignant lymphomas showed perforation, and adenocarcinomas showed perforation or bowel obstruction [11]. These symptoms are known to differ with the characteristics of the tumors. Gastrointestinal stromal tumors develop in the small bowel muscle layer and grow in the bowel submucosal layer. They may grow toward the lumen and compress it, or grow laterally, or grow as dumbbell shapes. Particularly, growth of the tumor toward the lumen causes necrosis and ulcers in the mucosal layer and impairs the blood supply to the small bowel mucosa, resulting in intestinal hemorrhage due to the necrosis in the mucosal membrane.

According to a study reported by Choi et al. [12] gastrointestinal stromal tumors occur preferentially in the jejunum, which has more abundant muscle layers than the ileum. Malignant lymphomas occur preferentially in the ileum because the lymphoid tissues called Peyer's patch associated with the intestine are distributed most abundantly overn the entire small intestine. The size of a malignant lymphoma is larger than $5 \mathrm{~cm}$ in most cases, and it may invade from the mucosa to the small bowel wall. Therefore, abdominal pain frequently develops, and intestinal perforation may occur in approximately $25 \%$ of the cases [13]. The causes of an adenocarcinoma can be diverse, such as genetic and environmental factors, but similar to stomach cancer or colorectal cancer, contact with carcinogenic materials may be an important factor. Therefore, adenocarcinomas have been reported to occur most preferentially in the duodenum, which has frequent contact with carcinogenic materials, followed by the jejunum [14].

An adenocarcinoma starts from a villous adenoma and progresses to malignancy due to several factors in some cases. In such cases, as the adenoma becomes larger, it may cause intestinal obstruction, and in some cases, hemorrhage and perforation caused by mucosal necrosis may be shown [15]. In our study, similar to other studies, gastrointestinal stromal tumors were observed to develop preferentially in the jejunum, and malignant lymphomas were observed to develop preferentially in the ileum $(P=0.001)$. In addition, in the analysis of the correlation of primary tumor site with major symptoms, tumors that developed in the jejunum showed hemorrhage most frequently, and tumors that developed in the ileum showed abdominal pain most frequently $(\mathrm{P}=0.048)$. This implies a correlation with histological distribution. While hemorrhage is the major symptom in the jejunum due to the high incidence of gastrointestinal stromal tumors, abdominal pain is mainly presented in the ileum due to the high incidence of malignant lymphomas.

Until now, the prognosis of malignant tumors in the small intestine has been known to be very poor. The major causes are nonspecific symptoms, delay of diagnosis due to the difficulty of diagnosis, high possibility of local metastasis or distant metastasis at the time of diagnosis, and detection of peritoneal metastasis and distant metastasis, up to $70 \%$, at the time of surgery [16]. Miles et al. [17] have reported the 5-year survival rate for all small bowel tumors to be 20-36. That of carcinoids was 73\%, which showed the best prognosis that of leiomyosarcomas was $29 \%$, that of malignant lymphomas was $27 \%$, and that of adenocarcinomas was $15 \%$, which showed the worst prognosis. The prognosis of small bowel adenocarcinomas is poor because of abundant lymphoid tissues in the small bowel mucosa, resulting in frequent local lymph node metastasis and liver metastasis. The 5-year survival rate was reported to be approximately $15-30 \%$ [18]. Lee et al. [19] have reported that the 5-year survival rate for primary small bowel lymphomas reached $53 \%$, and among patients who had been treated appropriately, $49.2 \%$ were completely cured.

Although numerous studies have been conducted on factors affecting the prognosis for gastrointestinal stromal tumors that develop in the small intestine, none of the factors has been established clearly. Gastrointestinal stromal tumors have diverse characteristics from benign to highly malignant. Therefore, gastrointestinal stromal tumors are frequently referred to as Stromal Tumors of Uncertain Malignant Potential [20]. Gastrointestinal stromal tumors in the small intestine have been reported to show recurrence rates higher than $50 \%$. Nevertheless, prognostic factors have not been established clearly, and whether the tumor is malignant or not can only be determined when local recurrence or metastasis occurs [21]. In our study, the 5 -year survival rate for malignant tumors was $64.8 \%$, and the 5-year survival rates for gastrointestinal stromal tumors, adenocarcinomas, and malignant lymphomas were $82.5 \%, 40 \%$, and $46.4 \%$, respectively, which were similar to other studies.

Although primary small bowel tumors are rare, such tumors represent a disease whose diagnosis may be delayed due to nonspecific symptoms. In our study, the symptoms of gastrointestinal stromal tumors, adenocarcinomas and malignant lymphomas occurring in the jejunum and the ileum differ according to the clinical characteristics, the location of the tumor, and the histological classification of the tumor. Therefore, in approaching small bowel tumor patients, although nonspecific, diagnostic approaches, while paying attention to the differences in symptoms according to clinical and histological classification, may be important for treatment and prognosis. 


\section{CONFLICT OF INTEREST}

No potential conflict of interest relevant to this article was reported.

\section{REFERENCES}

1. Herbsman H, Wetstein L, Rosen Y, Orces H, Alfonso AE, Iyer SK, et al. Tumors of the small intestine. Curr Probl Surg 1980; 17:121-82.

2. Yang YS, Huang QY, Wang WF, Sun G, Peng LH. Primary jejunoileal neoplasmas: a review of 60 cases. World J Gastroenterol 2003;9:862-4.

3. Cao J, Zuo Y, Lv F, Chen Z, Li J. Primary small intestinal malignant tumors: survival analysis of 48 postoperative patients. J Clin Gastroenterol 2008;42:167-73.

4. Catena F, Ansaloni L, Gazzotti F, Gagliardi S, Di Saverio S, De Cataldis A, et al. Small bowel tumours in emergency surgery: specificity of clinical presentation. ANZ J Surg 2005;75:997-9.

5. North JH, Pack MS. Malignant tumors of the small intestine: a review of 144 cases. Am Surg 2000;66:46-51.

6. Lowenfels AB. Why are small-bowel tumours so rare? Lancet 1973;1:24-6.

7. Calman KC. Why are small bowel tumours rare? An experimental model. Gut 1974;15:552-4.

8. Wattenberg LW. Studies of polycyclic hydrocarbon hydroxylases of the intestine possibly related to cancer: effect of diet on benzpyrene hydroxylase activity. Cancer 1971;28:99-102.

9. Darling RC, Welch CE. Tumors of the small intestine. N Engl J Med 1959;260:397-408.

10. Naef M, Buhlmann M, Baer HU. Small bowel tumors: diagnosis, therapy and prognostic factors. Langenbecks Arch Surg 1999;384: 176-80.
11. Blanchard DK, Budde JM, Hatch GF 3rd, Wertheimer-Hatch L, Hatch KF, Davis GB, et al. Tumors of the small intestine. World J Surg 2000;24:421-9.

12. Choi W, Chung JB, Song SY, Moon YM, Kang JK, Park IS. Primary neoplasms of the small bowel: how can we diagnose early? Korean J Gastroenterol 1996;28:632-40.

13. Jackson LN, Evers BM. Gastrointestinal lymphomas. In: Yeo CJ, editor. Shackelford's surgery of the alimentary tract. 6th ed. Vol. 1. Philadelphia: Elsevier; 2007. p. 1199-212.

14. Zouhairi ME, Venner A, Charabaty A, Pishvaian MJ. Small bowel adenocarcinoma. Curr Treat Options Oncol 2008;9:388-99.

15. Coco C, Rizzo G, Manno A, Mattana C, Verbo A. Surgical treatment of small bowel neoplasms. Eur Rev Med Pharmacol Sci 2010;14:327-33.

16. Ciccarelli O, Welch JP, Kent GG. Primary malignant tumors of the small bowel: the Hartford Hospital experience, 1969-1983. Am J Surg 1987;153:350-4.

17. Miles RM, Crawford D, Duras S. The small bowel tumor problem: an assessment based on a 20 year experience with 116 cases. Ann Surg 1979;189:732-40.

18. Dabaja BS, Suki D, Pro B, Bonnen M, Ajani J. Adenocarcinoma of the small bowel: presentation, prognostic factors, and outcome of 217 patients. Cancer 2004;101:518-26.

19. Lee J, Kim WS, Kim K, Ko YH, Kim JJ, Kim YH, et al. Intestinal lymphoma: exploration of the prognostic factors and the optimal treatment. Leuk Lymphoma 2004;45:339-44.

20. Pak JS, Kim BG, Cha SJ, Chang IT. Multiple gastrointestinal stromal tumors of the small intestine. J Korean Surg Soc 2005;69: 172-5.

21. Aparicio T, Boige V, Sabourin JC, Crenn P, Ducreux M, Le Cesne A, et al. Prognostic factors after surgery of primary resectable gastrointestinal stromal tumours. Eur J Surg Oncol 2004;30: 1098-103. 